\title{
How I Manage Cancer-Associated Thrombosis
}

\section{Management der Krebs-assoziierten Thrombose}

\author{
Florian Moik ${ }^{1}$ Cihan Ay ${ }^{1,2}$ \\ ${ }^{1}$ Clinical Division of Haematology and Haemostaseology, \\ Department of Internal Medicine I, Comprehensive Cancer Center \\ Vienna, Medical University of Vienna, Vienna, Austria \\ 2I.M. Sechenov First Moscow State Medical University (Sechenov \\ University), Moscow, Russia
}

Address for correspondence Cihan Ay, MD, Clinical Division of Hematology and Hemostaseology, Department of Medicine I, Medical University of Vienna, Waehringer Guertel 18-20, A-1090 Vienna, Austria (e-mail: cihan.ay@meduniwien.ac.at).

Hämostaseologie 2020;40:38-46.

\author{
Abstract \\ Keywords \\ - cancer \\ - thrombosis \\ - venous \\ thromboembolism \\ - anticoagulation \\ - DOAC
}

\section{Zusammenfassung}

Schlüssselwörter

- Krebs

- Thrombose

- venöse Thromboembolie

- Antikoagulation

- DOAK
In this concise review, we discuss some common clinical challenges in the management of patients with cancer-associated venous thromboembolism (VTE), a frequent complication in patients with cancer that increases morbidity and mortality. While direct oral anticoagulants (DOACs) have been established in clinical practice for anticoagulation in patients with VTE without cancer, their efficacy and safety in patients with cancer have not been assessed in randomized controlled trials until recently. The choice of the appropriate anticoagulant agent in the era of DOACs to treat patients with cancer-associated VTE is based on balancing the risk of recurrence against the risk of bleeding, and potential drug-drug interactions. However, the management of patients is challenged by special scenarios such as incidentally diagnosed pulmonary embolism and catheter-related thrombosis, and sometimes complicated by concomitant thrombocytopenia. We provide guidance for management of cancer-associated VTE in different clinical scenarios in a case-based manner and briefly review recent clinical studies and guidelines to explain our approach to management of the cases.

Im Rahmen dieser Übersichtsarbeit werden einige Facetten und Herausforderungen beleuchtet, die die Behandlung von PatientInnen mit Krebs-assoziierten venösen Thromboembolien (VTE) mit sich bringt. Während für die Behandlung der VTE bei PatientInnen ohne Krebs direkte orale Antikoagulanzien (DOAK) bereits ihren Einzug in die klinische Routine gefunden haben, waren Effektivität und Sicherheit dieser Medikament Therapie der Krebs-assoziierten VTE bis kürzlich nicht durch ausreichende Evidenz gesichert. Verkompliziert wird Antikoagulation bei Personen mit einer Krebserkrankung durch ein erhöhtes VTE-Rezidiv- und Blutungsrisiko, potentielle Medikamenteninteraktionen, eine begleitende Thrombozytopenie sowie spezielle klinische Fälle wie die Katheter-assoziierten Thrombose oder eine inzidentell diagnostizierte Pulmonalembolie. Das Ziel dieser Arbeit ist es, die neueste Evidenz zu den klinischen Herausforderungen einer Krebs-assoziierten VTE Fall-basiert zusammenzufassen und damit einen Leitfaden für den klinischen Alltag zu bieten. received

August 25, 2019

accepted after revision

November 4, 2019 (c) 2020 Georg Thieme Verlag KG Stuttgart · New York
DOI https://doi.org/

10.1055/s-0039-3402806.

ISSN 0720-9355. 


\section{Background}

Venous thromboembolism (VTE), a disease entity including deep venous thrombosis (DVT) and pulmonary embolism $(\mathrm{PE})$, is a frequent complication in patients with cancer, and increases morbidity, mortality, and health-care expenses. ${ }^{1-3}$ The risk of developing VTE is four to seven times higher for patients with cancer compared with the noncancer population, with cancer-associated VTE accounting for up to $18 \%$ of all VTE cases. ${ }^{1,4-6}$ Risk of VTE in patients with cancer strongly depends on underlying risk factors and varies according to cancer type, stage, treatment, and patient-related factors. ${ }^{7}$ Cumulative incidence rates of VTE in the general cancer population have been reported to be as high as $8 \%$ and reach $20 \%$ in specific cancer types such as brain, pancreatic, and stomach tumors. ${ }^{8}$ Managing VTE in the context of cancer involves several complex aspects and therefore can be challenging in clinical practice. Within this concise review we aim at summarizing up-to-date evidence on the treatment of cancer-associated VTE in a case-based manner and discuss some common clinical challenges.

\section{Case 1: A Patient with Active Lung Cancer and Symptomatic Venous Thromboembolism}

A 59-year-old man with non-small-cell lung cancer with metastasis to the contralateral lung presented to the emergency department 6 months after his initial diagnosis and after three cycles of chemoimmunotherapy (carboplatin/pemetrexed/ pembrolizumab). He reported progressive dyspnea and pain across the left hemithorax. Upon further questioning, he reported pain in the left calf for around 2 weeks. Diagnostic procedures were performed and found elevated $D$-dimer values $(15.0 \mu \mathrm{g} / \mathrm{mL}$, reference range: $<0.5 \mu \mathrm{g} / \mathrm{mL}$ ), a filling defect of a segmental artery of the left medial lobe on contrast-enhanced computed tomography (CT) of the chest as well as proximal DVT of the left lower extremity on ultrasonography. He reported no history of bleeding; his platelet count is $154 \mathrm{G} / \mathrm{L}$.

Evidence for Therapy of Acute Cancer-Associated VTE Until recently, patients with cancer-associated VTE have been recommended to be treated with low-molecular-weight hep$\operatorname{arin}(\mathrm{LMWH})$. This recommendation has been based on a lower risk of recurrent VTE compared with vitamin-K antagonists (VKA) in randomized controlled trials of LMWH versus VKA over a treatment duration of 3 to 6 months. ${ }^{9}$ The most relevant of these trials was the CLOT trial, which reported a significantly lower risk of recurrent VTE with LMWH compared with VKA (9 vs. $17 \%$ at 6 months; hazard ratio [HR]: $0.48 ; 95 \%$ confidence interval $[\mathrm{CI}]: 0.30-0.77 ; p=0.002$ ) and a similar risk of major bleeding ( 6 vs. $4 \%, p=0.27$ ). ${ }^{10}$

In contrast to VTE in noncancer patients, direct oral anticoagulants (DOACs) have not been established as standard treatment due to lack of robust evidence. In randomized controlled trials comparing DOAC to VKA for therapy of VTE, active cancer as an underlying risk factor has been mostly underrepresented and thereby subgroup analyses in this special cohort lacked power to confidently recommend their usage in this setting. ${ }^{11}$ Further, the standard treatment regimen in these studies was VKA, ${ }^{12-15}$ which was not the recommended treatment for VTE in the cancer population according to guidelines.

Recently, two phase III trials comparing DOACs to LMWH for treating cancer patients with acute VTE have been published. In the Hokusai VTE Cancer study, a prospective open-label, blinded endpoint evaluation (PROBE), noninferiority trial, patients were randomized to receive either LMWH for at least 5 days followed by oral edoxaban (60 mg once daily or in a reduced dose of $30 \mathrm{mg}$ once daily when creatinine clearance was below $50 \mathrm{~mL} / \mathrm{min}$, body weight of $<60 \mathrm{~kg}$, or concomitant use of a potent P-glycoprotein [P-gp] inhibitor) or subcutaneous dalteparin (200 international units [IU] per kilogram of body weight once daily for 1 month followed by $150 \mathrm{IU}$ per kilogram once daily). Treatment duration was 6 to 12 months. The composite outcome of recurrent VTE or major bleeding at 12 months of follow-up was observed in $12.8 \%$ in the edoxaban group and $13.5 \%$ in the dalteparin group (modified intention to treat population: 1,046 patients; HR: 0.97 [0.70-1.36]; $p=0.006$ for noninferiority). VTE occurred less frequently in the edoxaban group (7.9 vs. $11.3 \%$; difference in risk: $-3.4 \%$ [ -7.0 to 0.2 ]) but the incidence of major bleeding was higher (6.9 vs. $4.0 \%$; difference in risk: $2.9 \%$ [0.1-5.6]). The increased risk in major bleeding composed mostly of nonsevere upper gastrointestinal bleeding in patients with gastrointestinal malignancies, with no reported stratification according to exact type of gastrointestinal malignancy. ${ }^{16}$ In the SELECT-D trial, a multicenter, randomized, open-label, pilot trial compared the oral factor X inhibitor rivaroxaban ( $15 \mathrm{mg}$ twice daily for 3 weeks, then $20 \mathrm{mg}$ once daily for a total of 6 months) to dalteparin (200 IU $/ \mathrm{kg}$ daily during the first month, then 150 $\mathrm{IU} / \mathrm{kg}$ daily up to month 6). A total of 203 patients were randomized to each group. The primary efficacy outcome of 6 months' cumulative incidence of recurrent VTE was lower in the rivaroxaban group (4 vs. 11\%; HR: 0.43 [0.19-0.99]) compared with treatment with $\mathrm{LMWH}$, while rates of major bleeding were higher (6 vs. 4\%; HR: 1.83 [0.68-4.96]). Patients with upper gastrointestinal malignancies (esophagus or gastroesophageal junction) tended to experience major bleeding more frequently in the rivaroxaban group (4/11 patients [36\%] vs. $1 / 19$ patients [11\%]). Rates of clinically relevant nonmajor bleeding (CRNMB) were higher in the rivaroxaban group (13 vs. 4\%; HR: 3.76 [1.63-8.69]) comprising mostly of gastrointestinal and urothelial bleeding in patients with colorectal and bladder cancer, respectively. ${ }^{17}$ One single-center experience with the use of rivaroxaban in patients with cancer and acute VTE, excluding patients with active gastrointestinal or genitourinary lesions, revealed promising efficacy and safety with cumulative incidence rates of recurrent VTE, major bleeding, and CRNMB of $4.2,2.2$, and $5.5 \%$, respectively $(n=1,072) .{ }^{18}$ For both the Hokusai VTE cancer trial and the SELECT-D trial, no excess in intracranial bleeding has been reported for the DOAC and the control group (Hokusai VTE cancer: edoxaban: 2 events [intracranial bleeding] in 522 patients, dalteparin: 4 events in 522 patients; SELECT-D: no intracranial bleeding in both treatment groups). ${ }^{16,17}$ 


\section{Discussion of Management}

The two phase III studies evaluating the use of DOACs versus LMWH have established edoxaban and rivaroxaban as alternatives to LMWH in the treatment for cancer-associated VTE. However, prior to deciding on a certain anticoagulation modality to treat patients in clinical practice, certain factors have to be considered.

First, the patient preference has to be respected in the sense of informed shared decision making. Further, efficacy and safety have to be balanced according to underlying additional risk factors such as an increased risk of bleeding, history of bleeding, renal function, or the presence of thrombocytopenia. According to ISTH SSC (International Society on Thrombosis and Hemostasis, Scientific and Standardization Committee) guidance, DOACs are suggested not to be used as first choice treatment in patients with VTE and luminal gastrointestinal malignancies (esophageal, gastric, or colorectal cancer with an intact primary), risk of bleeding from the genitourinary tract or nephrostomy tubes, and active gastrointestinal mucosal abnormalities (e.g., duodenal ulcers, gastritis, colitis, esophagitis). ${ }^{19}$ This suggestion is based on the higher risk of bleeding in patients treated with a DOAC compared with LMWH in the HOKUSAI VTE Cancer and SELECT-D trials. Further, when selecting the type of anticoagulation, factors like adequate dosing, renal function, and potential drug-drug interactions have to be considered. Especially DOACs, as of their metabolization via the cytochrome P450 3A4 enzyme (CYP3A4) and their transport via P-gp, harbor the risk of being influenced by concomitantly administered drugs. A variety of anticancer drugs or supportive care medication such as certain antiemetics and fungostatics interfere with CYP3A4 and/or P-gp and might thereby have an impact on efficacy and safety. ${ }^{20}$ The application of LMWH is a valid alternative in patients in whom strong interactions are suspected. In the Hokusai VTE Cancer trial, special attention was paid to the interaction with strong P-gp inhibitors/inducers as patients treated with protease inhibitors have been excluded from the study and doses of edoxaban were reduced to $30 \mathrm{mg}$ daily in patients treated with certain tyrosine kinase inhibitors, hormonal agents, immunomodulatory drugs, and azol antifungals for the time of concomitant application. ${ }^{16}$

The use of VKA does not play a role in the initial treatment of cancer-associated thrombosis, due an unfavorable outcome profile compared with LMWH. ${ }^{10}$ VKAs also exhibit many drug-drug interaction potentials, and require regular monitoring and dose adjustment, especially in patients receiving chemotherapy. However, they are still an alternative, if both DOACs and LMWHs are not feasible or available.

Despite lack of robust evidence, the treatment duration is usually recommended to be 3 to 6 months, with an extended use beyond 6 months for patients with ongoing active malignancy, as these patients are at an elevated risk for recurrent VTE compared with patients with cured cancer or cancer in remission. ${ }^{21-24}$

In summary, the use of edoxaban or rivaroxaban is suggested in patients with cancer and acute VTE when there is a low underlying risk of bleeding and no potential drugdrug interaction ( - Fig. 1) ${ }^{19}$

\section{Management of Case 1}

This 59-year old patient with active cancer undergoing chemotherapy had no history of bleeding and no additional risk factors for bleeding. His platelet count was in the normal range and the renal function was not impaired. The patient was informed about therapeutic options and especially the low risk of intracranial bleeding and a higher risk of gastrointestinal bleeding with DOAC were explained to him. Therefore, in accordance to the patient's preference (favoring oral application over subcutaneous injections), anticoagulation with rivaroxaban $15 \mathrm{mg}$ twice daily after 3 days of therapeutic anticoagulation with LMWH was initiated. After 21 days rivaroxaban was reduced to $20 \mathrm{mg}$ once daily according to drug labeling. During anticoagulation with rivaroxaban, the renal function and platelet counts were regularly monitored. After 6 months, a follow-up visit to decide whether to terminate anticoagulation has been scheduled. Unfortunately, after initially responding to chemotherapy, his cancer has progressed in the meantime and he was switched to second-line chemotherapy. He experienced no recurrent episode of VTE and no bleeding events during anticoagulation. In this case of active malignancy and ongoing chemotherapy, anticoagulation was continued, due to a high risk of VTE recurrence. ${ }^{25,26}$ It was recommended to continue rivaroxaban at a dose of $20 \mathrm{mg}$ once daily with regular monitoring of kidney function parameters and platelet counts. However, this recommendation is based on expert opinion as the evidence for the extended treatment for secondary prevention of VTE in patients with cancer is relatively poor.

\section{Case 2: A Patient with Breast Cancer, Symptomatic Deep Vein Thrombosis, and Low Platelet Counts}

A 45-year old female patient with stage IIb breast cancer was undergoing primary induction chemotherapy with fluorouracil, etoposide, and cyclophosphamide. Several days after her last cycle of chemotherapy, she complained about pain in her left calf. Clinical evaluation revealed unilateral swelling in the affected leg with pitting edema and distension of superficial veins. Compression ultrasonography revealed obstruction of the left common femoral vein. The patient had no history of VTE or bleeding and a normal renal function. However, her blood counts revealed grade 2 thrombocytopenia, with a platelet count of $64 \mathrm{G} / \mathrm{L}$.

\section{Evidence for Therapy of VTE in a Patient with Thrombocytopenia}

Thrombocytopenia is a common consequence of chemotherapy and increases the risk of bleeding. Interestingly, despite low platelet counts the risk of VTE in a patient with cancer remains high. ${ }^{8,27}$ The risk of recurrent VTE strongly depends on the presence of risk factors such as type of malignancy with reported rates of 10 to $44 \%$, and rates of bleeding range between 7 and 33\% in patients with cancer-associated thrombosis and thrombocytopenia. ${ }^{28,29} \mathrm{~A}$ threshold of $50 \mathrm{G} / \mathrm{L}$ of platelets has been recommended for dosing of therapeutic anticoagulation. Above this threshold, full-dose anticoagulation has been reported to be safe and is recommended by the ISTH SSC. ${ }^{29,30}$ For 


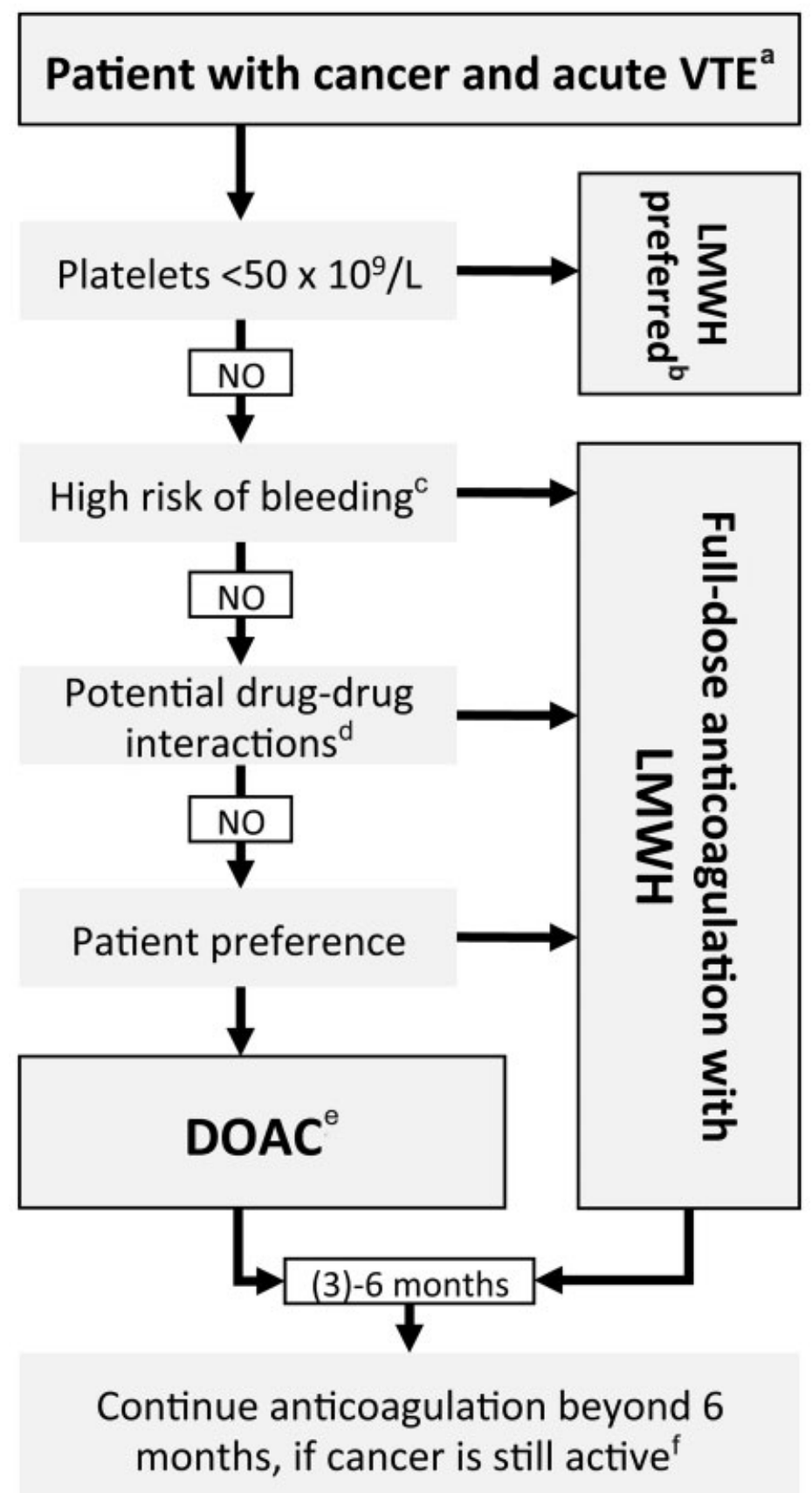

Fig. 1 Treatment algorithm for patients with acute cancer-associated VTE according to ISTH SSC 2018 guidance statement. ${ }^{a}$ Venous thrombosis and/or pulmonary embolism. ${ }^{\mathrm{b}} \mathrm{LMWH}$ dosing according to platelet count and risk of thrombus progression according to Fig. $2 .{ }^{\mathrm{C}} \mathrm{High}$ risk of bleeding includes luminal $\mathrm{Gl}$ cancer with primary in place, cancer at risk of bleeding from GU-tract or nephrostomy tubes, and active $\mathrm{Gl}$ mucosal abnormalities (gastric/duodenal ulcers, gastritis, esophagitis, colitis). Interactions with drugs that influence CYP3A4 and/or P-gp. ${ }^{e}$ Rivaroxaban monotherapy or edoxaban monotherapy after LMWH lead-in. ${ }^{f}$ Active cancer: cancer that has not been surgically removed (active tumor burden in patient); antineoplastic treatment is being administered; hematological cancer not in complete remission. CYP3A4,

cytochrome P450 3A4; DOAC, direct oral anticoagulant; GI, gastrointestinal; GU, genitourinary; ISTH, International Society on Thrombosis and Haemostasis; LMWH, low-molecular-weight heparin; P-gp, P-glycoprotein; SSC, Scientific and Standardization Committee; VTE, venous thromboembolism.

patients with platelet counts below $50 \mathrm{G} / \mathrm{L}$, anticoagulation can either (1) be applied in full dose with concomitant platelet transfusion or (2) in reduced doses preferably with a $\mathrm{LMWH}^{30}$ The guidance for this challenging clinical situation of anticoagulation in a patient with cancer-associated thrombosis and thrombocytopenia is based on retrospective studies and small prospective observational cohort studies, as randomized trials comparing different agents and dosing regimens in this setting are lacking. ${ }^{31-34}$ For patients in the acute phase of VTE $(\leq 30$ days since the event) and high-risk features for recurrence such as symptomatic, proximal PE, proximal DVT, or history of previous thrombotic events, therapeutic dosing of LMWH with platelet transfusion to maintain platelet counts above 40 to $50 \mathrm{G} / \mathrm{L}$ is suggested. ${ }^{30}$ For patients with a lower risk of 
recurrence (incidental, subsegmental $\mathrm{PE}$, catheter-related thrombosis [CRT], distal DVT) or patients in the chronic and subacute phases of cancer-associated VTE ( $>30$ days since the event), dose adjustment (50\%, or therapeutic dose, or prophylactic dose) of LMWH is suggested. ${ }^{30}$ In general, anticoagulation should be withheld for patients with platelet counts $<25 \mathrm{G} / \mathrm{L}$ and resumed when platelets increase again, with full therapeutic anticoagulation being possible when platelet counts are $>50 \mathrm{G} / \mathrm{L}^{30}$ The use of DOACs in patients with severe thrombocytopenia $(<50 \mathrm{G} / \mathrm{L})$ is not recommended, because no data are available to support their use in this setting and there is potential increase of bleeding risk with DOAC compared with LMWH. ${ }^{16,17,30}$ In patients with acute VTE and absolute contraindications to anticoagulation, for example in patients with active bleeding or those with severe, prolonged thrombocytopenia when platelet transfusion is not possible or not efficacious to increase the platelet count, the use of a retrievable inferior vena cava filter might be considered on a case-by-case basis. $^{30,35,36}$

In summary, patients with platelet counts above $50 \mathrm{G} / \mathrm{L}$ should be treated with full-dose anticoagulation, either
LMWH or DOACs. For patients with severe thrombocytopenia (platelet count $<25 \mathrm{G} / \mathrm{L}$ ), anticoagulation should be paused. For those between 25 and $50 \mathrm{G} / \mathrm{L}$, anticoagulation should be tailored according to individual risk of thrombus progression and timing after diagnosis of the thrombotic event with adjusted dose (half the therapeutic dose or prophylactic dose of LMWH) or in full dose after platelet transfusion to maintain the platelet count above 40 to $50 \mathrm{G} / \mathrm{L}$. - Fig. 2 describes an algorithm for the management of patients with cancer-associated VTE and concomitant thrombocytopenia.

\section{Management of Case 2}

In this patient with mild thrombocytopenia, full-dose anticoagulation is acceptable, as suggested by the guidance of the ISTH SSC and suggested in the protocol of both the SELECT-D and the Hokusai VTE cancer study. After 5 days of therapeutic dose of LMWH, edoxaban at a dose of $60 \mathrm{mg}$ once daily was prescribed. She did not experience recurrent VTE or bleeding episodes, with close monitoring of her blood counts over the course of her chemotherapy treatment.

\section{Patient with cancer-associated VTE and thrombocytopenia}

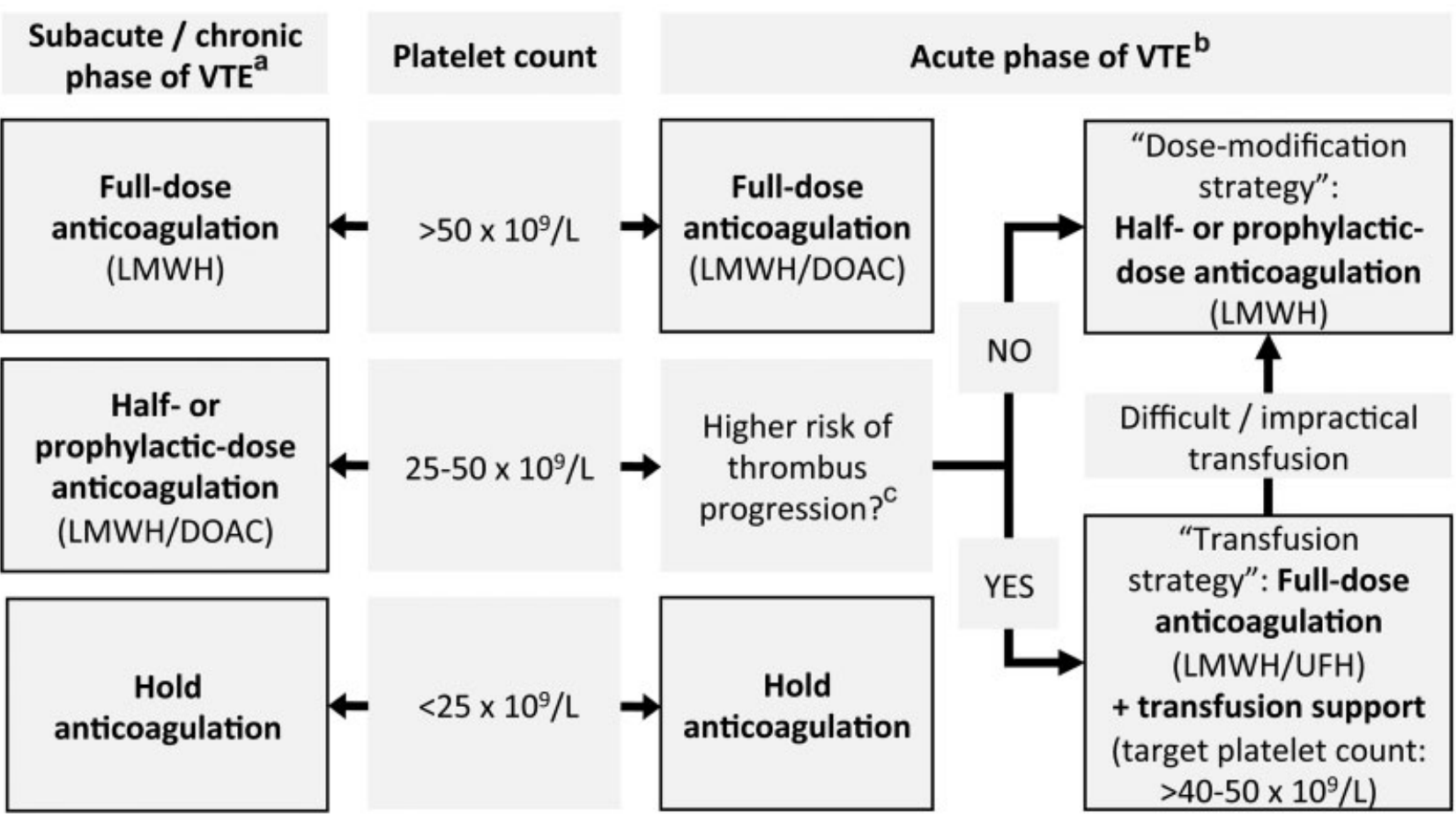

Retrievable IVC-filters:

Case-by-case basis in acute VTE event and (1) active bleeding or (2) severe, prolonged thrombocytopenia when anticoagulation with platelet transfusion support cannot be achieved ${ }^{d}$

\footnotetext{
Fig. 2 Management of cancer-associated thrombosis in patients with thrombocytopenia according to ISTH SSC 2018 guidance statement.

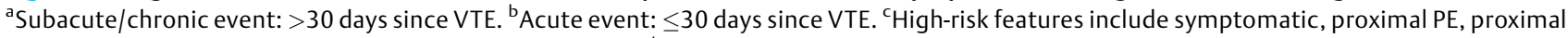
DVT, or history of previous/recurrent thrombotic events. 'IVC filters should only be considered in patients with absolute contraindication to anticoagulation and be removed as soon as anticoagulation is resumed. DOAC, direct oral anticoagulant; DVT, deep vein thrombosis; ISTH, International Society on Thrombosis and Haemostasis; IVC, inferior vena cava; LMWH, low-molecular-weight heparin; PE, pulmonary embolism; SSC, Scientific and Standardization Committee; UFH, unfractionated heparin; VTE, venous thromboembolism.
} 
After 3 months, the patient underwent curative surgery, with perioperative discontinuation of edoxaban, which was stopped 48 hours prior to surgery and restarted on the third day postsurgery. Due to the high risk of VTE in patients undergoing major cancer surgery, thromboprophylaxis with LMWH was initiated in the evening of the surgery and continued until the restart of edoxaban. ${ }^{37,38}$ At a follow-up visit after 6 months, the patient was in remission after curative surgical treatment and had no further anticancer therapy. Therefore, the decision to terminate anticoagulation was made.

\section{Case 3: A Patient with Cancer and Incidental Pulmonary Embolism}

A 67-year old male patient with recurrent gastric cancer metastatic to the liver has been currently undergoing chemotherapy (cisplatin/5-fluorouracil). After four cycles of chemotherapy the patient underwent contrast enhanced CT for restaging purposes. The patient's cancer was stable in comparison to baseline imaging. However, a filling defect of a segmental branch of the right upper pulmonary artery was detected. On thorough questioning, the patient reported no symptoms suggestive of either PE and/or DVT. On compression ultrasonography of both lower extremities, no evidence of thrombotic occlusion was detected. The patient has had a normal renal function, grade 1 thrombocytopenia $(120 \mathrm{G} / \mathrm{L})$, and no history of bleeding. The treating oncologist asked the hematologist for consultation on how to manage the patient and whether to commence anticoagulation or not.

\section{Management of a Patient with Cancer and Incidental Pulmonary Embolism}

Incidentally diagnosed VTE in patients with cancer, detected mostly on restaging imaging procedures, is a common issue in oncologic practice. Up to $5 \%$ of patients with cancer will develop incidental VTE during the course of their disease and around 50\% of all cancer-associated VTE is estimated to be detected incidentally. ${ }^{39,40}$ Based on findings of several retrospective cohort studies, international guidelines suggest the same therapeutic approach for incidental VTE as for symptomatic events. ${ }^{21,23,41}$ For example, a retrospective cohort study by den Exter et al, comparing outcomes of incidental with symptomatic VTE in patients with cancer, found similar recurrence rates (13.3 vs. $16.9 \%) .{ }^{42}$ Also Sahut et al reported similar rates of recurrence for incidental and symptomatic events (6.1 vs. $7.7 \%$ ) in another retrospective study. ${ }^{43}$ In a large, multicenter prospective cohort study, rates of recurrence were found to be 6\% (4.4-81) during 12 months of follow-up. ${ }^{44}$ A recent subgroup analysis of the Hokusai VTE cancer trial revealed similar rates of recurrence and bleeding in patients with incidental and those with symptomatic VTE. ${ }^{45}$ Overall, $53 \%$ of patients included in the SELECT-D study and 33\% of patients in the Hokusai VTE cancer trial had asymptomatic index events. ${ }^{16,17}$ In contrast, previous studies comparing the efficacy and safety of LMWH versus VKA did not include patients with incidental VTE events. ${ }^{10}$
The clinical management of incidentally diagnosed asymptomatic subsegmental PE still is a matter of debate. In a general cohort of cancer and noncancer patients, higher detection rates of subsegmental PE on multidetector CT compared with single-detector CT did not lower the risk of recurrent VTE, as reported by a systematic review conducted by Carrier et al. ${ }^{46}$ Further, a retrospective study investigating outcomes of incidental, subsegmental, and more proximal locations of PE found an increased risk of death in patients with PE located in segmental and more proximal branches of pulmonary arteries, while subsegmental PE was not associated with an increased risk of mortality. ${ }^{47}$ However, a pooled analysis of retrospective studies ${ }^{48}$ and a prospective cohort study found comparable risk of recurrent thrombosis in patients with cancer-associated VTE for subsegmental and more proximal PEs (6.4 vs. $6.0 \%, p=0.93) .{ }^{44}$ Based on these findings, the ISTH SSC formulated several suggestions on the management of incidental PE. First, radiological findings should be reviewed by an experienced radiologist. After confirmation of the diagnosis of incidental, subsegmental PE, bilateral compression ultrasonography of both legs should be performed to evaluate potentially concomitant DVT. Patients who are found to have proximal DVT are suggested to receive therapeutic anticoagulation. For the remainder of patients with isolated, incidental subsegmental PE, with or without asymptomatic distal DVT, therapeutic decision-making should balance individual risk of recurrence and bleeding, with short-interval clinical follow-up recommended for patients who are decided not to receive anticoagulation and compression ultrasonography after 1 week in patients with concomitant DVT. ${ }^{41}$ - Fig. 3 describes an algorithm of clinical management of patients with cancer and incidental PE based on these recommendations.

\section{Management of Case 4}

In this patient with active cancer and asymptomatic incidental segmental PE, therapeutic anticoagulation was indicated. As of the elevated risk of bleeding in patients with upper gastrointestinal malignancies when treated with DOACs, the use of LMWH is favorable in this setting and was recommended to the patient. His mild thrombocytopenia should be closely monitored but does not necessitate dose adjustment at the time.

\section{Case 4: A Patient with Symptomatic Catheter-Related Thrombosis}

A 71-year old woman with rectal cancer is currently undergoing induction chemoradiotherapy. After two cycles of therapy, administered via right-sided peripherally inserted central catheter (PICC), her treating physician notices diffuse swelling of her entire right arm and pitting edema. Upon questioning, the patient reports intermittent pain in the affected arm since 3 days and no chest pain, dyspnea, and hemoptysis are present. Ultrasonography reveals obstruction of the axillary and subclavian veins. She has no history of bleeding, her renal function is within the normal range, and her platelet count is $89 \mathrm{G} / \mathrm{L}$. There are no signs of infection. 


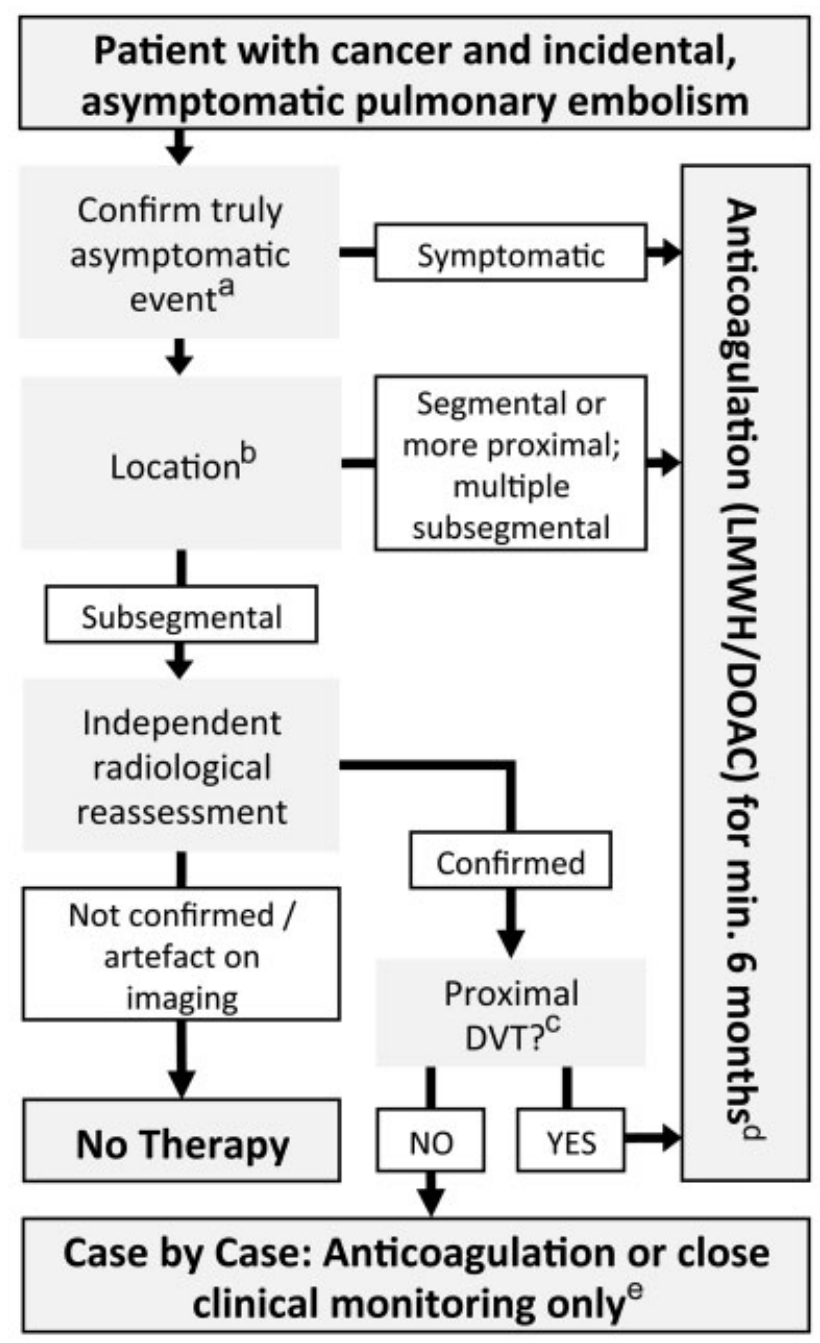

Fig. 3 Management of incidental, asymptomatic PE modified according to ISTH SSC 2015 guidance statement. ${ }^{a}$ Careful review of history and clinical examination is recommended to exclude symp-

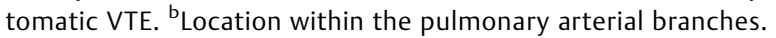
${ }^{\mathrm{c}}$ Evaluation of concomitant asymptomatic DVT on bilateral compression ultrasonography. ${ }^{\mathrm{d}}$ Therapeutic anticoagulation recommendations extrapolated from data on symptomatic events according to - Fig. 1. ${ }^{e}$ Considering risk of bleeding, risk factors for recurrent thrombosis, performance status, patient preference; clinical monitoring + serial bilateral compression ultrasonography after 1 week for patients with distal DVT. DOAC, direct oral anticoagulants; DVT, deep vein thrombosis; ISTH, International Society on Thrombosis and Haemostasis; LMWH, low-molecular-weight heparin; PE: pulmonary embolism; SSC, Scientific and Standardization Committee; VTE, venous thromboembolism.

\section{Evidence on Therapeutic Management}

Patients with cancer often receive antineoplastic or supportive therapy via central venous catheters (CVCs). This local trigger, influencing blood flow, in combination with a systemic state of hypercoagulability due to the underlying malignancy, harbors the risk of CRT. Reported incidence rates of CRT vary widely, with rates of asymptomatic events of up to $50 \%$ and symptomatic events of 1 to $5 \%$ in a general population of patients with $\mathrm{CVC}^{49}{ }^{49}$ In a prospective cohort study by Lee et al, the cumulative incidence of CRT in patients with cancer was $4 \%$ after a median of 30 days after inser- tion, ${ }^{50}$ and in the prospective study by Decousus et al a 3.8\% incidence of CRT was found after 12 months of follow-up. ${ }^{51}$ The risk factors for CRT were, amongst others, type of CVC (highest in PICC), size of catheter in relation to the inserted vein, position of catheter tip, placement via the jugular vein, history of VTE, systemic or local inflammation, and metastatic cancer. ${ }^{49}$

Management of CRT is a "grey zone" due to lack of highquality evidence. Expert guidance suggests leaving the catheter in situ, given the absence of local inflammation, malfunction, or incorrect positioning. Therapeutic anticoagulation, preferably LMWH, should be initiated with a proposed duration of treatment of 3 to 6 months or longer if the catheter is still in place. ${ }^{52,53}$

Recommendations on modality of therapeutic anticoagulation are mostly based on extrapolations of data from clinical trials on cancer-associated DVT/PE and on observational studies. In a systematic review on treatment of CRT, Baumann Kreuziger et al reported rates of recurrent VTE of $7.0 \%$ and rates of major bleeding of 2.8 to $4.9 \%$ of anticoagulated patients, with large variations in treatment modality and duration. ${ }^{54}$ In a prospective pilot study, which investigated the outcome of anticoagulation with VKA in 74 patients with cancer and CRT, no recurrent VTE and four major bleeding events were reported. ${ }^{55}$ However, based on data on management of cancerassociated VTE in general, LMWH is usually recommended over VKA. ${ }^{21,52}$ The application of DOACs in the setting of CRT remains a matter of discussion. In a prospective pilot study conducted by Davies et al, evaluating the use of rivaroxaban in 80 patients with cancer and CRT, rates of recurrent VTE and major bleeding were $1.43 \%(0.25-7.66)$ and $12.85 \%$ (6.9-22.7), respectively. ${ }^{56}$

In summary, patients with CRT should receive therapeutic anticoagulation for 3 to 6 months or longer, if the catheter is still in place. The CVC can be left in situ, if no signs of infection are present, it is functional and still in use. LMWH is suggested as the first choice for anticoagulation, with only limited data on the use of DOACs in this setting.

\section{Management of Case 4}

In this patient with rectal cancer and CRT, anticoagulation was initiated with full-dose LMWH. Her catheter was functional and left in place. Symptoms of CRT have resolved during anticoagulant treatment. After 5 months, the patient had undergone curative resection and received adjuvant chemotherapy via her CVC. The patient was in complete remission and the decision to remove her PICC was made, because no further chemotherapy was planned. The patient did not experience recurrent VTE or bleeding complications. Due to the absence of ongoing prothrombotic risk factors, the decision to terminate anticoagulation was made.

\section{Summary and Conclusion}

Clinical management of patients with cancer-associated VTE is challenging. Balancing the risk of recurrence with risk of bleeding and patient preferences in the age of emerging evidence for DOAC needs a personalized approach to select 
the appropriate treatment. Special clinical cases such as incidentally diagnosed PE, DVT of the upper extremity in association with a CVC, and the presence of thrombocytopenia are frequent scenarios that further complicate the management of patients. In general, according to recent publications and guidelines, DOACs are an effective and safe alternative to LMWH in patients with cancer-associated VTE. However, in certain subpopulations, e.g., in patients with gastrointestinal tumors, risk of gastrointestinal bleeding is higher with DOAC compared with LMWH. Further research is needed to better stratify risk of bleeding and VTE recurrence, to evaluate the impact of drug-drug interactions and to provide data on other DOACs, as for apixaban, which is currently being investigated within the CARAVAGGIO trial. ${ }^{57}$ Recommendations on special clinical scenarios are often based on low evidence and more data are needed to produce robust evidence and thereby improve management of anticoagulation cancer patients with incidental PE, CRT, and thrombocytopenia.

\section{Conflict of Interest}

Dr. Ay reports personal fees from Bayer, personal fees from Daiichi-Sankyo, and personal fees from BMS/Pfizer, outside the submitted work.

\section{References}

1 Walker AJ, Card TR, West J, Crooks C, Grainge MJ. Incidence of venous thromboembolism in patients with cancer-a cohort study using linked United Kingdom databases. Eur J Cancer 2013;49 (06):1404-1413

2 Khorana AA, Francis CW, Culakova E, Kuderer NM, Lyman GH. Thromboembolism is a leading cause of death in cancer patients receiving outpatient chemotherapy. J Thromb Haemost 2007;5 (03):632-634

3 Kourlaba G, Relakis J, Mylonas C, et al. The humanistic and economic burden of venous thromboembolism in cancer patients: a systematic review. Blood Coagul Fibrinolysis 2015; 26(01):13-31

4 Heit JA, Silverstein MD, Mohr DN, Petterson TM, O'Fallon WM, Melton LJ III. Risk factors for deep vein thrombosis and pulmonary embolism: a population-based case-control study. Arch Intern Med 2000;160(06):809-815

5 Heit JA, O'Fallon WM, Petterson TM, et al. Relative impact of risk factors for deep vein thrombosis and pulmonary embolism: a population-based study. Arch Intern Med 2002;162(11): $1245-1248$

6 Blom JW, Doggen CJ, Osanto S, Rosendaal FR. Malignancies, prothrombotic mutations, and the risk of venous thrombosis. JAMA 2005;293(06):715-722

7 Ay C, Pabinger I, Cohen AT. Cancer-associated venous thromboembolism: Burden, mechanisms, and management. Thromb Haemost 2017;117(02):219-230

8 Timp JF, Braekkan SK, Versteeg HH, Cannegieter SC. Epidemiology of cancer-associated venous thrombosis. Blood 2013;122(10): $1712-1723$

9 Posch F, Königsbrügge O, Zielinski C, Pabinger I, Ay C. Treatment of venous thromboembolism in patients with cancer: a network meta-analysis comparing efficacy and safety of anticoagulants. Thromb Res 2015;136(03):582-589

10 Lee AYY, Levine MN, Baker RI, et al; Randomized Comparison of Low-Molecular-Weight Heparin versus Oral Anticoagulant Therapy for the Prevention of Recurrent Venous Thromboembolism in
Patients with Cancer (CLOT) Investigators. Low-molecular-weight heparin versus a coumarin for the prevention of recurrent venous thromboembolism in patients with cancer. N Engl J Med 2003;349 (02):146-153

11 Ay C, Kamphuisen PW, Agnelli G. Antithrombotic therapy for prophylaxis and treatment of venous thromboembolism in patients with cancer: review of the literature on current practice and emerging options. ESMO Open 2017;2(02):e000188

12 Prins MH, Lensing AW, Brighton TA, et al. Oral rivaroxaban versus enoxaparin with vitamin $\mathrm{K}$ antagonist for the treatment of symptomatic venous thromboembolism in patients with cancer (EINSTEIN-DVT and EINSTEIN-PE): a pooled subgroup analysis of two randomised controlled trials. Lancet Haematol 2014;1(01): e37-e46

13 Agnelli G, Buller HR, Cohen A, et al. Oral apixaban for the treatment of venous thromboembolism in cancer patients: results from the AMPLIFY trial. J Thromb Haemost 2015;13(12): 2187-2191

14 Raskob GE, van Es N, Segers A, et al; Hokusai-VTE investigators. Edoxaban for venous thromboembolism in patients with cancer: results from a non-inferiority subgroup analysis of the HokusaiVTE randomised, double-blind, double-dummy trial. Lancet Haematol 2016;3(08):e379-e387

15 Schulman S, Goldhaber SZ, Kearon C, et al. Treatment with dabigatran or warfarin in patients with venous thromboembolism and cancer. Thromb Haemost 2015;114(01):150-157

16 Raskob GE, van Es N, Verhamme P, et al; Hokusai VTE Cancer Investigators. Edoxaban for the treatment of cancer-associated venous thromboembolism. N Engl J Med 2018;378(07):615-624

17 Young AM, Marshall A, Thirlwall J, et al. Comparison of an oral factor Xa inhibitor with low molecular weight heparin in patients with cancer with venous thromboembolism: results of a randomized trial (SELECT-D). J Clin Oncol 2018;36(20):2017-2023

18 Soff GA, Mones J, Wilkins C, et al. Rivaroxaban treatment of cancer-associated venous thromboembolism: Memorial Sloan Kettering Cancer Center institutional experience. Res Pract Thromb Haemost 2019;3(03):349-356

19 Khorana AA, Noble S, Lee AYY, et al. Role of direct oral anticoagulants in the treatment of cancer-associated venous thromboembolism: guidance from the SSC of the ISTH. J Thromb Haemost 2018;16(09):1891-1894

20 Gelosa P, Castiglioni L, Tenconi M, et al. Pharmacokinetic drug interactions of the non-vitamin $\mathrm{K}$ antagonist oral anticoagulants (NOACs). Pharmacol Res 2018;135:60-79

21 Kearon C, Akl EA, Ornelas J, et al. Antithrombotic therapy for VTE disease: CHEST guideline and expert panel report. Chest 2016; 149(02):315-352

22 Farge D, Debourdeau P, Beckers M, et al. International clinical practice guidelines for the treatment and prophylaxis of venous thromboembolism in patients with cancer. J Thromb Haemost 2013;11(01):56-70

23 Lyman GH, Bohlke K, Khorana AA, et al; American Society of Clinical Oncology. Venous thromboembolism prophylaxis and treatment in patients with cancer: american society of clinical oncology clinical practice guideline update 2014. J Clin Oncol 2015;33(06):654-656

24 van der Hulle T, den Exter PL, van den Hoven P, et al. Cohort study on the management of cancer-associated venous thromboembolism aimed at the safety of stopping anticoagulant therapy in patients cured of cancer. Chest 2016;149(05):1245-1251

25 Francis CW, Kessler CM, Goldhaber SZ, et al. Treatment of venous thromboembolism in cancer patients with dalteparin for up to 12 months: the DALTECAN Study. J Thromb Haemost 2015;13(06): 1028-1035

26 Jara-Palomares L, Solier-Lopez A, Elias-Hernandez T, et al. Tinzaparin in cancer associated thrombosis beyond 6months: TiCAT study. Thromb Res 2017;157:90-96 
27 Slichter SJ, Kaufman RM, Assmann SF, et al. Dose of prophylactic platelet transfusions and prevention of hemorrhage. N Engl J Med 2010;362(07):600-613

28 Kopolovic I, Lee AY, Wu C. Management and outcomes of cancerassociated venous thromboembolism in patients with concomitant thrombocytopenia: a retrospective cohort study. Ann Hematol 2015;94(02):329-336

29 Khanal N, Bociek RG, Chen B, et al. Venous thromboembolism in patients with hematologic malignancy and thrombocytopenia. Am J Hematol 2016;91(11):E468-E472

30 Samuelson Bannow BT, Lee A, Khorana AA, et al. Management of cancer-associated thrombosis in patients with thrombocytopenia: guidance from the SSC of the ISTH. J Thromb Haemost 2018; 16(06):1246-1249

31 Campbell PM, Ippoliti C, Parmar S. Safety of anticoagulation in thrombocytopenic patients with hematologic malignancies: a case series. J Oncol Pharm Pract 2017;23(03):220-225

32 Mantha S, Miao Y, Wills J, Parameswaran R, Soff GA. Enoxaparin dose reduction for thrombocytopenia in patients with cancer: a quality assessment study. J Thromb Thrombolysis 2017;43(04): 514-518

33 Monreal M, Zacharski L, Jiménez JA, Roncales J, Vilaseca B. Fixeddose low-molecular-weight heparin for secondary prevention of venous thromboembolism in patients with disseminated cancer: a prospective cohort study. J Thromb Haemost 2004;2(08): $1311-1315$

34 Pemmaraju N, Kroll MH, Afshar-Kharghan V, Oo TH. Bleeding risk in thrombocytopenic cancer patients with venous thromboembolism (VTE) receiving anticoagulation. Blood 2012;120(21): 3408

35 Brunson A, Ho G, White R, Wun T. Inferior vena cava filters in patients with cancer and venous thromboembolism (VTE): patterns of use and outcomes. Thromb Res 2016;140(Suppl 1): S132-S141

36 Kearon C, Akl EA, Comerota AJ, et al. Antithrombotic therapy for VTE disease: antithrombotic therapy and prevention of thrombosis, 9th ed: American College of Chest Physicians evidence-based clinical practice guidelines. Chest 2012;141(02, Suppl):e419S-e496S

37 Steffel J, Verhamme P, Potpara TS, et al; ESC Scientific Document Group. The 2018 European Heart Rhythm Association practical guide on the use of non-vitamin K antagonist oral anticoagulants in patients with atrial fibrillation. Eur Heart J 2018;39(16): 1330-1393

38 Key NS, Khorana AA, Kuderer NM, et al. Venous thromboembolism prophylaxis and treatment in patients with cancer: ASCO Clinical Practice Guideline Update. J Clin Oncol 2019 (e-pub ahead of print). 10.1200/JCO.19.01461. Published online August 05, 2019

39 van Es N, Bleker SM, Di Nisio M. Cancer-associated unsuspected pulmonary embolism. Thromb Res 2014;133(Suppl 2):S172-S178

40 Di Nisio M, Carrier M. Incidental venous thromboembolism: is anticoagulation indicated? Hematology (Am Soc Hematol Educ Program) 2017;2017(01):121-127

41 Di Nisio M, Lee AYY, Carrier M, Liebman HA, Khorana AA; Subcommittee on Haemostasis and Malignancy. Diagnosis and treatment of incidental venous thromboembolism in cancer patients: guidance from the SSC of the ISTH. J Thromb Haemost 2015;13(05):880-883

42 den Exter PL, Hooijer J, Dekkers OM, Huisman MV. Risk of recurrent venous thromboembolism and mortality in patients with cancer incidentally diagnosed with pulmonary embolism: a comparison with symptomatic patients. J Clin Oncol 2011;29(17): 2405-2409

43 Sahut D'Izarn M, Caumont Prim A, Planquette B, et al. Risk factors and clinical outcome of unsuspected pulmonary embolism in cancer patients: a case-control study. J Thromb Haemost 2012;10 (10):2032-2038

44 Kraaijpoel N, Bleker SM, Meyer G, et al; UPE investigators. Treatment and long-term clinical outcomes of incidental pulmonary embolism in patients with cancer: an international prospective cohort study. J Clin Oncol 2019;37(20):1713-1720

45 Mulder FI, van Es N, Kraaijpoel N, et al. Efficacy and safety of edoxaban in clinically relevant subgroups: results from the Hokusai VTE Cancer randomized trial. Thromb Res 2018;164:S194

46 Carrier M, Righini M, Wells PS, et al. Subsegmental pulmonary embolism diagnosed by computed tomography: incidence and clinical implications. A systematic review and meta-analysis of the management outcome studies. J Thromb Haemost 2010;8 (08):1716-1722

47 O'Connell C, Razavi P, Ghalichi M, et al. Unsuspected pulmonary emboli adversely impact survival in patients with cancer undergoing routine staging multi-row detector computed tomography scanning. J Thromb Haemost 2011;9(02):305-311

48 van der Hulle T, den Exter PL, Planquette B, et al. Risk of recurrent venous thromboembolism and major hemorrhage in cancerassociated incidental pulmonary embolism among treated and untreated patients: a pooled analysis of 926 patients. J Thromb Haemost 2016;14(01):105-113

49 Geerts W. Central venous catheter-related thrombosis. Hematology (Am Soc Hematol Educ Program) 2014;2014(01):306-311

50 Lee AYY, Levine MN, Butler G, et al. Incidence, risk factors, and outcomes of catheter-related thrombosis in adult patients with cancer. J Clin Oncol 2006;24(09):1404-1408

51 Decousus H, Bourmaud A, Fournel P, et al; ONCOCIP Investigators. Cancer-associated thrombosis in patients with implanted ports: a prospective multicenter French cohort study (ONCOCIP). Blood 2018;132(07):707-716

52 Zwicker JI, Connolly G, Carrier M, Kamphuisen PW, Lee AYY. Catheter-associated deep vein thrombosis of the upper extremity in cancer patients: guidance from the SSC of the ISTH. J Thromb Haemost 2014;12(05):796-800

53 Debourdeau P, Farge D, Beckers M, et al. International clinical practice guidelines for the treatment and prophylaxis of thrombosis associated with central venous catheters in patients with cancer. J Thromb Haemost 2013;11(01):71-80

54 Baumann Kreuziger L, Onwuemene O, Kolesar E, Crowther M, Lim W. Systematic review of anticoagulant treatment of catheterrelated thrombosis. Thromb Res 2015;136(06):1103-1109

55 Kovacs MJ, Kahn SR, Rodger M, et al. A pilot study of central venous catheter survival in cancer patients using low-molecular-weight heparin (dalteparin) and warfarin without catheter removal for the treatment of upper extremity deep vein thrombosis (The Catheter Study). J Thromb Haemost 2007;5(08):1650-1653

56 Davies GA, Lazo-Langner A, Gandara E, et al. A prospective study of Rivaroxaban for central venous catheter associated upper extremity deep vein thrombosis in cancer patients (Catheter 2). Thromb Res 2018;162:88-92

57 Agnelli G, Becattini C, Bauersachs R, et al; Caravaggio Study Investigators. Apixaban versus dalteparin for the treatment of acute venous thromboembolism in patients with cancer: The Caravaggio Study. Thromb Haemost 2018;118(09):1668-1678 